PSFC/JA-00-38

\title{
Pedestal Profiles and Measurements in C-Mod Enhanced D-alpha H-modes
}

\author{
A.E. Hubbard, R.L. Boivin, B.A. Carreras ${ }^{1}$, R.S. Granetz, \\ J.W. Hughes, I.H. Hutchinson, J. Irby, V. Klein ${ }^{2}$, B. LaBombard, \\ Y. Lin, E.S. Marmar, A. Mazurenko, D. Mossessian, \\ E. Nelson-Melby, T. Sunn Pedersen ${ }^{3}$, M. Porkolab, J.A. Snipes, \\ J. Terry, S. Wolfe
}

December 2000

\author{
Plasma Science and Fusion Center \\ Massachusetts Institute of Technology \\ Cambridge, MA 02139 USA
}

\footnotetext{
${ }^{1}$ Oak Ridge National Laboratory, Oak Ridge, TN, 37831

${ }^{2}$ Univ. New Mexico, Albuquerque, NM 87106

${ }^{3}$ Columbia Univ., New York, NY
}

This work was supported by the U.S. Department of Energy, Cooperative Grant No. DE-FC02-99ER54512. Reproduction, translation, publication, use and disposal, in whole or in part, by or for the United States government is permitted.

Submitted for publication to Physics of Plasmas. 


\title{
Pedestal profiles and fluctuations in C-Mod enhanced D-alpha H-modes
}

A.E. Hubbard, R. L. Boivin, B. A. Carreras*, R.S. Granetz, M. Greenwald, J. W. Hughes, I.H. Hutchinson, J. Irby, V. Klein", B. LaBombard, Y. Lin, E.S. Marmar, A. Mazurenko, D. Mossessian, E. Nelson-Melby, T. Sunn Pedersen ${ }^{\circledR}$, M. Porkolab, J. A. Snipes, J. Terry, S. Wolfe, S. Wukitch

Plasma Science and Fusion Center, MIT, Cambridge MA, 02129

*Oak Ridge National Laboratory, Oak Ridge, TN, 37831

${ }^{\#}$ Univ. New Mexico, Albuquerque, NM 87106

${ }^{\circledR}$ Columbia Univ., New York, NY

\begin{abstract}
High resolution measurements on the Alcator C-Mod tokamak [I.H. Hutchinson et al, Phys. Plasmas 1, 1551 (1994)] of the transport barrier in the "Enhanced $\mathrm{D}_{\alpha}$ " (EDA) regime, which has increased particle transport without large edge localized modes, show steep density and temperature gradients over a region of $2-5 \mathrm{~mm}$, with peak pressure gradients up to 12 $\mathrm{MPa} / \mathrm{m}$. Evolution of the pedestal at the LH transition is consistent with a large, rapid drop in thermal conductivity across the barrier. A quasi-coherent fluctuation in density, potential and $\mathrm{B}_{\text {pol }}$, with $\mathrm{f}_{\mathrm{o}} \sim 50-150 \mathrm{kHz}$ and $\mathrm{k}_{\theta} \sim 4 \mathrm{~cm}^{-1}$, always appears in the barrier during EDA, and drives a large particle flux. Conditions to access the steady-state EDA regime in deuterium include $\delta>$ $0.35, \mathrm{q}_{95}>3.5$ and L-mode target density $\overline{\mathrm{n}}_{\mathrm{e}}>1.2 \times 10^{20} \mathrm{~m}^{-3}$. A reduced $\mathrm{q}_{95}$ limit is found for hydrogen discharges.
\end{abstract}




\section{Introduction}

This paper reports on recent edge measurements on the Alcator C-Mod tokamak (CMod), primarily in the "Enhanced $\mathrm{D}_{\alpha}$ ", or EDA, H-mode regime. The "high confinement", or "H-mode" regime, first discovered on the Axisymmetric Divertor Experiment (ASDEX) in $1982^{1}$, is characterized by a decrease in particle and energy transport, and consequent steepening of density and temperature gradients, in a narrow region just inside the last closed flux surface (LCFS). In recent years it has become apparent that the plasma parameters at the top of the transport barrier, or 'pedestal', can strongly affect global confinement, not only increasing the stored energy, W, by the height of the pedestal but also modifying the boundary conditions of core transport. Several experiments, notably C-Mod and ASDEX-Upgrade ${ }^{2,3,4}$, have shown correlations between edge $\mathrm{T}_{\mathrm{e}}$ and core confinement and core $\nabla \mathrm{T}$, or between edge pressure and W. Such sensitivity has led to experimental and theoretical efforts to understand the physics and scaling of the edge pedestal ${ }^{5}$, and to increase pedestal temperature and pressure.

Unfortunately, there is a serious negative consequence to increasing pedestal energy. In most high power experiments, the edge pressure is regulated by Type I Edge Localized Modes (ELMs) ${ }^{6}$. The edge gradients collapse periodically due to a magnetohydrodynamic (MHD) instability, expelling much of the energy in the pedestal to the scrape-off-layer (SOL). This energy, which can be $5-10 \%$ percent of the total $\mathrm{W}$, is rapidly transported to the divertor plates. Extrapolations to a reactor-grade plasma such as the International Tokamak Experimental Reactor (ITER) show that the resulting heat pulse would cause unacceptably high erosion of divertor plates ${ }^{7}$. From a divertor standpoint, smaller, more frequent ELMs such as Type III ELMs are preferable. However, these seem to occur when edge $T_{e}$, and thus confinement, are only moderately above L-mode.

The Enhanced $D_{\alpha}$ regime found on C-Mod may satisfy the conflicting requirements of high confinement and divertor heat loads. In this regime the pedestal is regulated by a continuous transport process, rather than by periodic MHD events. First observed in $1996^{8}$, it is characterized by much lower particle confinement than ELM-free H-mode, leading to steadystate conditions and lower radiated power ${ }^{9}$. Energy confinement is only moderately reduced, and is comparable to that with Type I ELMs on other devices. Regular Type I ELMs have not yet been observed on C-Mod. Since the EDA regime is very attractive for a reactor, understanding its cause and the conditions under which it occurs and characterizing the edge and global 
parameters in EDA H-mode are important aims of the C-Mod experimental program. Due to the compact size $(\mathrm{a}=0.21 \mathrm{~m}, \mathrm{R}=0.68 \mathrm{~m})$ and high field and density $\left(\mathrm{B}_{\mathrm{T}} \leq 8 \mathrm{~T}, \overline{\mathrm{n}}_{\mathrm{e}} \leq 10^{21} \mathrm{~m}^{-3}\right)$ of $\mathrm{C}$ Mod, the device occupies a unique parameter space and has very narrow pedestals, with widths $\Delta$ $<1 \mathrm{~cm}^{10}$. Much of the recent progress in understanding the EDA regime has come from improved measurements of the profiles and fluctuations in the pedestal region.

In the next section, the steady-state profiles and time evolution of pedestal parameters, primarily $\mathrm{n}_{\mathrm{e}}$ and $\mathrm{T}_{\mathrm{e}}$, are presented. Since the results turn out to be similar in ELM-free and EDA H-modes, both regimes are covered. Section III gives detailed observations in the EDA regime, in particular of quasi-coherent fluctuations which are found to cause the enhanced particle transport. The conditions to access the regime are examined. Possible physical explanations for the fluctuations are discussed in Section IV.

\section{Pedestal characteristics and evolution}

\section{A. Diagnostics}

Several high resolution diagnostics have been added to measure both pedestal profiles and fluctuations. Of particular importance is a new high resolution edge Thomson scattering (TS) diagnostic ${ }^{11}$, which samples the plasma vertically at up to 22 spatial points, giving radial resolution $\delta R$ of $1.5 \mathrm{~mm}$ when mapped to the midplane, and measures $\mathrm{T}_{\mathrm{e}}$ and $\mathrm{n}_{\mathrm{e}}$ every $33 \mathrm{~ms}$. $\mathrm{T}_{\mathrm{e}}$ profiles are also measured with two electron cyclotron emission (ECE) polychromators at the midplane, which have lower spatial resolution but sub-ms time resolution ${ }^{10}$. The density pedestal can also be inferred from a CCD (charge coupled device) array measuring visible bremsstrahlung ${ }^{12}$, proportional to $\mathrm{n}^{2} \mathrm{Z}_{\mathrm{eff}} f\left(\mathrm{~T}_{\mathrm{e}}\right)$, which has $\delta \mathrm{t}=1 \mathrm{~ms}$ and $\delta \mathrm{R}=1 \mathrm{~mm}$. Two soft $\mathrm{x}-$ ray arrays, with $\delta \mathrm{R}=1.5 \mathrm{~mm}$, are mainly sensitive to pedestals in the density of medium $\mathrm{Z}$ impurities, primarily fluorine. ${ }^{13}$ High resolution diagnostics of XUV and deuterium Lyman $\alpha$ emission enable the photon radiation and local neutral density and ionization rates to be measured $^{14,15}$.

Edge fluctuation diagnostics include a reflectometer measuring density fluctuations at 5 critical density layers between 0.3 and $1.5 \times 10^{20} \mathrm{~m}^{-3}$, which are typically all in or near the pedestal region ${ }^{16}$. Phase contrast imaging (PCI), viewing vertically and integrating along vertical chords, is able to resolve fluctuation wavenumbers along the major radius using 12 channels with $3 \mathrm{~mm}$ radial spacing. Two scanning Langmuir probes measure fluctuations in density and 
potential, as well as edge $n_{e}$ and $T_{e}$ profiles. For some discharges a magnetic fluctuation probe was added to the midplane probe head.

\section{B. Pedestal parameters}

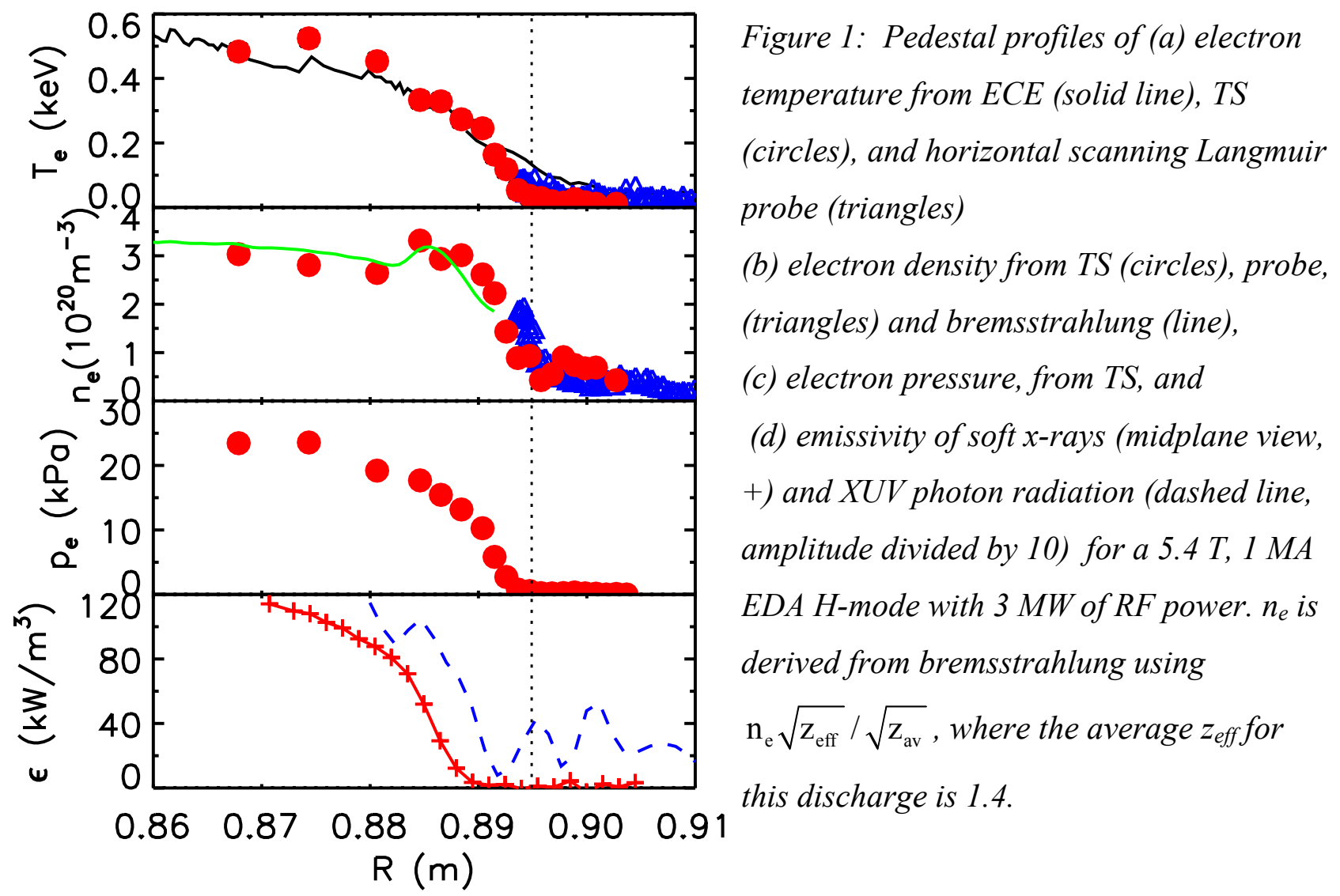

An example of pedestal profiles in $T_{e}, n_{e}, p_{e}$ and soft $x$-ray and XUV emissivity, obtained in an RF heated EDA H-mode discharge, is shown in Fig.1. In order to compare data from various diagnostics, all parameters are mapped to the outer midplane. Uncertainties in flux reconstruction using $\mathrm{EFIT}^{17}$, and in diagnostic positions, give rise to relative errors $\sim 5 \mathrm{~mm}$. It can be seen that all plasma parameters vary sharply over only a few $\mathrm{mm}$, with $\mathrm{n}_{\mathrm{e}}$ and impurity densities having the narrowest widths. The steepest pressure and $\mathrm{T}_{\mathrm{e}}$ gradients, exceeding 10 $\mathrm{MPa} / \mathrm{m}$ and $60 \mathrm{keV} / \mathrm{m}$ respectively, occur in the outer part of the pedestal, where $\mathrm{n}_{\mathrm{e}}$ also drops sharply. At this point $T_{e}$ is less than $200 \mathrm{eV}$, so that the collisionality $v^{*}$ is typically $5-10$. Inside this region $T_{e}$ continues to increase for several $\mathrm{mm}$ with a lower gradient of $\sim 25 \mathrm{keV} / \mathrm{m}$, but still above the core $\nabla \mathrm{T}_{\mathrm{e}}$ of $\sim 10 \mathrm{keV} / \mathrm{m}$. The transition between edge and core gradients, which sets the boundary condition for core confinement, typically occurs between $\psi=0.90$ and 0.95 , where 
$\psi$ is the normalized poloidal magnetic flux. ECE measurements have shown that this region of improved $\nabla \mathrm{T}_{\mathrm{e}}$ expands inward with increasing net power $\mathrm{P}_{\text {in }}-\mathrm{P}_{\text {rad }}{ }^{4}$; the outer region of steepest $\nabla \mathrm{T}_{\mathrm{e}}$ does not expand. In contrast, the density pedestal is independent of power. In order to characterize the height and width of the pedestals in a systematic way and compare with other experiments, profiles are fit with a tanh function ${ }^{18}$. However, such functions cannot capture all of the structure revealed by TS profiles; fitted widths tend to depend on the exact region of the plasma fitted as well as diagnostic resolution. This may well account for some of the data scatter and discrepancies noted in scalings using different diagnostics, and between different experiments ${ }^{5}$.

Scalings of pedestal parameters in RF-heated H-modes have been examined in a range of plasma conditions, $\mathrm{B}_{\mathrm{T}}=4.5-6 \mathrm{~T}, \mathrm{I}_{\mathrm{p}}=0.6-1.2 \mathrm{MA}, \mathrm{P}_{\mathrm{RF}}=1-5 \mathrm{MW}$ and line averaged density $\overline{\mathrm{n}}_{\mathrm{e}}=$ 1-6 $\times 10^{20} \mathrm{~m}^{-3}$. The heights of the $\mathrm{T}_{\mathrm{e}}$ and $\mathrm{n}_{\mathrm{e}}$ pedestals show fairly clear dependences. As might be expected, $\mathrm{n}_{\mathrm{e}, \text { ped }}$ is proportional to $\overline{\mathrm{n}}_{\mathrm{e}}$. The edge temperature at $\psi=0.9$ scales as $\left(\mathrm{P}_{\mathrm{in}} / \overline{\mathrm{n}}_{\mathrm{e}}\right)^{0.5 \pm 0.1}$ at fixed current, and also increases with $I_{p}$. There is a strong correlation between $I_{p}$ and both $n_{e, p e d}$ and $\bar{n}_{\mathrm{e}}$ in H-mode. The pedestal pressure thus also increases with current. The dependence is mainly in the pressure gradient $\nabla \mathrm{p}_{\mathrm{e}}$, rather than pedestal width. The best empirical scaling found for this is $\nabla \mathrm{p}_{\mathrm{e}} \propto \mathrm{I}_{\mathrm{p}}^{1.24 \pm 0.08} \boldsymbol{V}_{\text {ped }}^{-0.37 \pm 0.03}$. Interestingly, none of the electron pedestal widths shows a clear dependence on $\mathrm{I}_{\mathrm{p}}$. Widths do not get systematically narrower, in contrast to scalings with $\rho_{\text {pol }}, \beta_{\text {pol }}^{0.4}$ or $\rho_{\text {pol }}^{0.6}$ which have been reported on the Japan Tokamak-60 Upgrade (JT-60U) and DIII-D experiments ${ }^{19,20}$, but consistent with measurements on ASDEX Upgrade ${ }^{21}$. As has been reported previously, the width of the x-ray pedestals does decrease with $\mathrm{I}_{\mathrm{p}}^{22}$. Our present understanding is that this reflects the width of the impurity barrier, formed near the top of the density pedestal as a result of neoclassical pinches, and that it depends on the particle diffusivity ${ }^{13}$. The outboard $\mathrm{x}$-ray width is also the only one to show a systematic difference between ELM-free and EDA plasmas. It is wider in EDA, consistent with the higher observed impurity particle transport ${ }^{22} . \mathrm{T}_{\mathrm{e}}$ and $\mathrm{n}_{\mathrm{e}}$ pedestal profiles in ELM-free and EDA H-modes with similar global parameters are indistinguishable.

The stability of ideal infinite-n ballooning modes in the pedestal has been analysed using TS profiles, assuming $T_{i}$ is equal to $T_{e}$ to derive the total pressure. For a discharge similar to 
that shown in Fig. 1 which had $\nabla \mathrm{P}$ up to $12 \mathrm{MPa} / \mathrm{m}$, near the maximum observed to date, the gradient is found to exceed the first stability limit calculated using only ohmic currents, by about $40 \%$. However, when the collisional edge bootstrap current is included in EFIT reconstructions, the limit increases and a narrow region of apparent second stable access appears. The pedestal is then found to be stable to ideal ballooning.

\section{Time evolution}

Since C-Mod uses ion cyclotron resonance heating (ICRH), which has continuously variable power, and the edge parameters are measured with millisecond time resolution, the time evolution of the H-mode pedestal can be followed both at the L-H and H-L transitions and as a function of power. Figure 2 illustrates the behaviour of $\mathrm{T}_{\mathrm{e}}$ and $\mathrm{n}_{\mathrm{e}}$, both at $\mathrm{r} / \mathrm{a}=0.86$ which is inboard of the edge transport barrier, for an EDA H-mode discharge in which $\mathrm{P}_{\mathrm{RF}}$ was slowly ramped from 0.5-2.0 MW and then down to 0. The top trace shows a jump in $\mathrm{T}_{\text {edge }}$ between Lmode and $\mathrm{H}$-mode, on a few ms time scale, as well as a moderate dependence on power in each regime. This clearly shows the bifurcation in thermal transport. Strong hysteresis between the L$\mathrm{H}$ and $\mathrm{H}-\mathrm{L}$ power thresholds can be seen. As previously found on C-Mod, data are consistent with a threshold in edge temperature, or a closely related quantity, to enter and remain in $\mathrm{H}$ mode $^{23}$. The density, in contrast, also shows a bifurcation at the L-H and H-L transitions but does not vary with power in each regime. Since the fuelling comes only from edge ionization, which depends on edge profiles, both particle transport and sources are different in L and H$\operatorname{mode}^{15}$. 


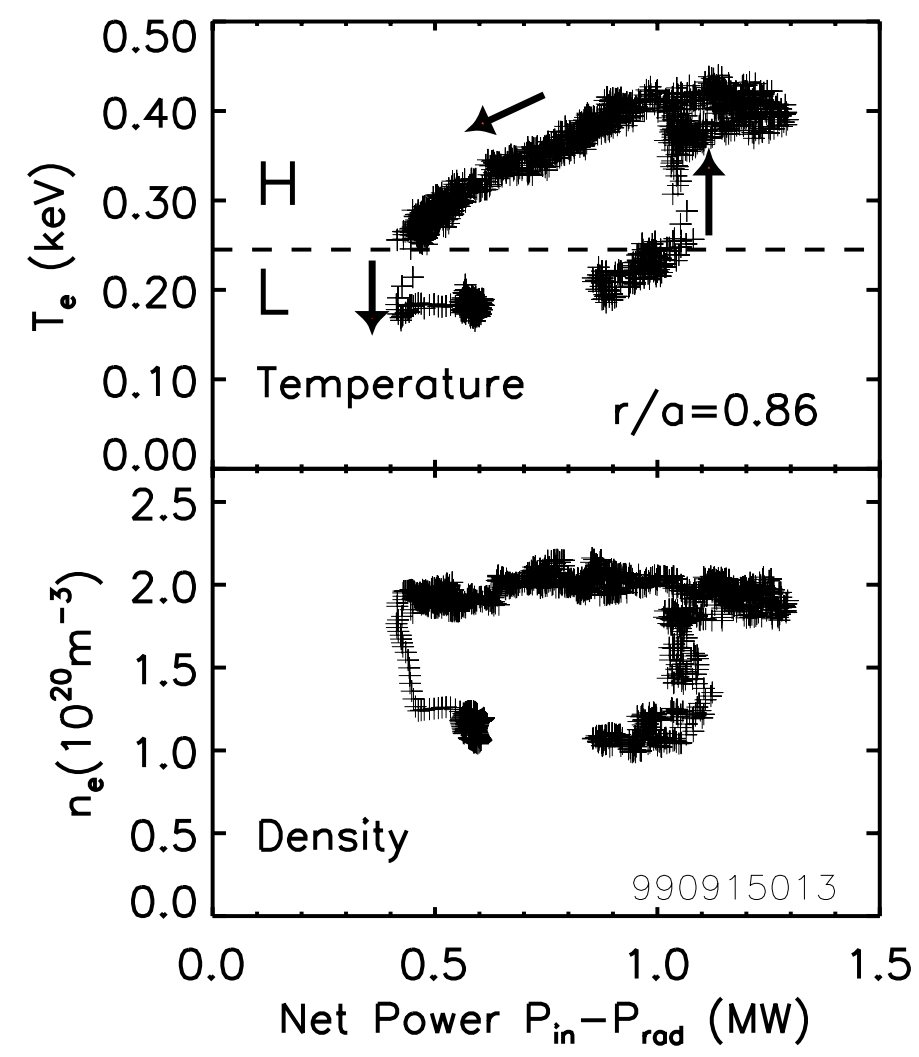

Figure 2: Variation of $T_{e}($ top $)$ and $n_{e}$ (bottom) at r $/ a=0.86$ vs net power $P_{i n}-P_{r a d}$, at 0.5 ms intervals during $a$ single 5.4 T, 0.8 MA discharge with slowly varied RF power. Arrows indicate the direction of time evolution. Points above the dashed line are in H-mode, which is EDA after the first few ms.

The fast evolution at the $\mathrm{L}-\mathrm{H}$ transition of a similar discharge with a power ramp is shown in Figure 3(a). Both edge $T_{e}$ and $n_{e}$ rise sharply, with initial rates of $\sim 20 \mathrm{keV} / \mathrm{s}$ and 2.3 $\mathrm{x} 10^{21} \mathrm{~m}^{-3} / \mathrm{s}$ respectively. All ECE channels in the edge region show a simultaneous break in slope, within the experimental determination of $0.5 \mathrm{~ms}$; the rate of increase is largest near the top of the pedestal. Variation of $\mathrm{dP} / \mathrm{dt}$ from $0.6 \mathrm{MW} / \mathrm{s}$ to $11 \mathrm{MW} / \mathrm{s}$ did not give a systematic variation of $\mathrm{dT}_{\mathrm{e}} / \mathrm{dt}$, indicating that the edge $\mathrm{T}_{\mathrm{e}}$ is not a unique function of the incremental power P-P $\mathrm{P}_{\text {thresh. }}$. Rather, the rate of increase in energy is consistent with a sudden drop of $\chi_{\mathrm{e}}$ across the transport barrier, so that the whole input power $\mathrm{P}_{\text {thresh }}$ is effectively blocked and contributes to the rapid pedestal formation. 

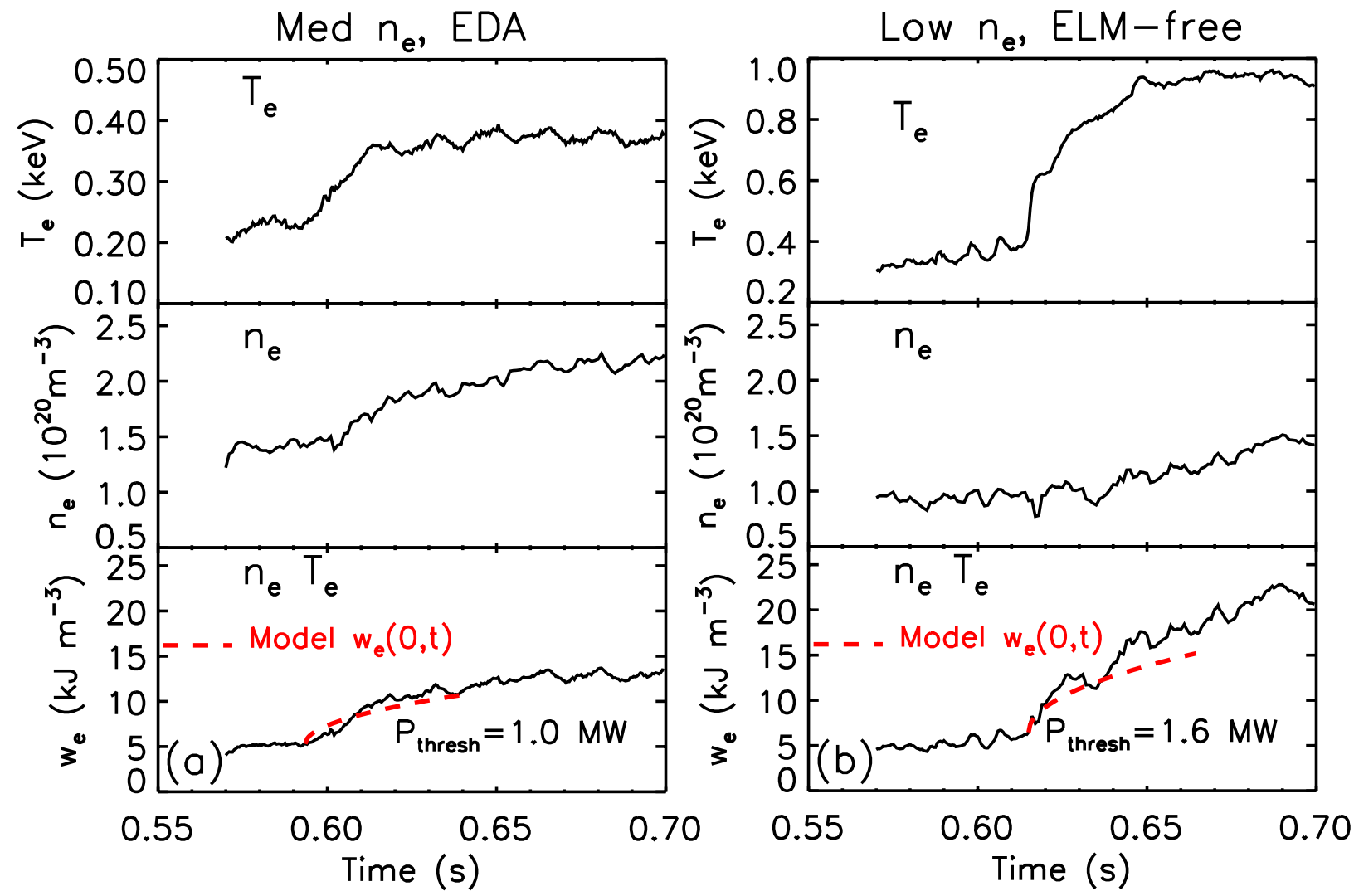

Figure 3: (a) Evolution of $T_{e}$ (top trace), $n_{e}$ (middle trace) and $w_{e}=\mathrm{k} n_{e} T_{e}$, measured at $r / a=$ 0.88 , at the L-H transition for a 5.4 T, 0.8 MA EDA H-mode discharge with $\overline{\mathrm{n}}_{\mathrm{e}}=1.8 \times 10^{20}$ $m^{-3}$ in L-mode. The dashed line in the bottom panel shows the prediction of Eq. 1, taking $\chi_{A}=$ $0.5 \mathrm{~m}^{2} / \mathrm{s}$ and $\chi_{b}=0.02 \mathrm{~m}^{2} / \mathrm{s}$.

(b) Same parameters, for a discharge identical except that L-mode $\overline{\mathrm{n}}_{\mathrm{e}}=1.18 \times 10^{20} \mathrm{~m}^{-3}$, which remains ELM-free. Note the higher $T_{e}$ scale. The same $\chi s$ are used to predict $\Delta w_{e}(t)$.

The evolution of the energy pedestal which would result from a decrease in transport from an anomalous diffusion coefficient $\chi_{\mathrm{A}}$ to a reduced level $\chi_{\mathrm{b}}$ in the barrier (eg $\chi_{\text {neo }}$ ) has been modelled both numerically and analytically. For constant power flux $\mathrm{q}_{\mathrm{in}}$, the greatest change occurs at the time of the transition and at the inside of the region of decreased transport, where $\mathrm{dw}_{\text {ped }} / \mathrm{dt}=\mathrm{q}_{\text {in }}-\mathrm{q}_{\text {out }}=\left(1-\chi_{\mathrm{b}} / \chi_{\mathrm{A}}\right) \mathrm{q}_{\text {in }}$ and $\mathrm{w}_{\text {ped }}$ is the total plasma energy at the pedestal top. Its evolution after the transition $(t=0)$ is given by 


$$
w_{p e d}(t)=w_{p e d}(0)+\frac{q\left(\chi_{A}-\chi_{b}\right)}{\sqrt{\pi}\left(\chi_{b}^{1 / 2}+\chi_{A}^{1 / 2}\right) \chi_{A}} \sqrt{t}
$$

The predicted rates of change at other positions are slower, and have a more complicated time dependence.

The thick solid line in the lower trace of Fig. 3(a) shows the prediction of Eq. 1, taking $\chi_{\mathrm{A}}$ $=0.5 \mathrm{~m}^{2} / \mathrm{s}$, determined from an L-mode power balance analysis, and $\chi_{\mathrm{b}}=0.02 \mathrm{~m}^{2} / \mathrm{s}$. This simple model agrees quite well with the measured $\mathrm{w}_{\text {ped }}$, which does have an approximately $\mathrm{t}^{1 / 2}$ dependence. The evolution of a lower density, ELM-free discharge is shown in Fig. 3(b) and is again fairly well described by Eq. 1, taking the same conductivities; the experimental $\mathrm{w}_{\text {ped }}$ is slightly higher. However, the relative contributions of $T_{e}$ and $n_{e}$ are very different. At low density the $\mathrm{T}_{\mathrm{e}}$ transient clearly dominates and the density rise is 8 times weaker, $3 \times 10^{20} \mathrm{~m}^{-3} / \mathrm{s}$. This reflects variation in the particle source available to fuel the H-mode at low density. The total ionization rate derived from Lyman $\alpha$ measurements is also about an order of magnitude lower, $\sim 5 \times 10^{20} / \mathrm{s}$. At higher density, with a source rate of $\sim 6 \times 10^{21} / \mathrm{s}$, the two terms in the pressure increase are comparable. A more complete transport analysis of the transient behaviour at other radii and plasma conditions will be presented in a separate publication.

\section{EDA H-mode}

\section{A. Global Characteristics}

The defining global feature of EDA H-modes is that they have much lower particle confinement, both of the main species and of impurities, than ELM-free H-modes. The lower $\tau_{\mathrm{p}}$ in EDA has been shown most clearly by impurity ablation experiments ${ }^{24}$, and is also reflected by a decrease in the rates of rise of both density and radiated power. $\mathrm{n}_{\mathrm{e}}$ and $\mathrm{P}_{\text {rad }}$ usually reach a steady state, as shown in Fig. 4 . Steady phases of up to $\sim 8 \tau_{\mathrm{E}}$ have been achieved, limited only by the duration of the plasma discharge and the RF power. The change in $\tau_{\mathrm{p}}$ is usually accompanied by an increase in the $\mathrm{D}_{\alpha}$ emission to a level comparable to L-mode, leading to the name of the regime. Energy confinement in EDA H-mode is also lower than in ELM-free Hmodes, but by a much smaller fraction than the particle confinement. The H-factor, defined as the ratio of $\tau_{\mathrm{E}}$ to the H-mode scaling $\tau_{\mathrm{ITER} 89-\mathrm{P}}{ }^{25}$, can be up to 2 , with $\sim 1.8$ being more typical. $\tau_{\mathrm{E}}$ is consistent with the ELMy H-mode scaling, ITER98y $2^{26}$. The major advantage of the EDA 
regime over Type I ELMy H-mode is that the increased particle transport occurs by a continuous, rather than an intermittent, process.

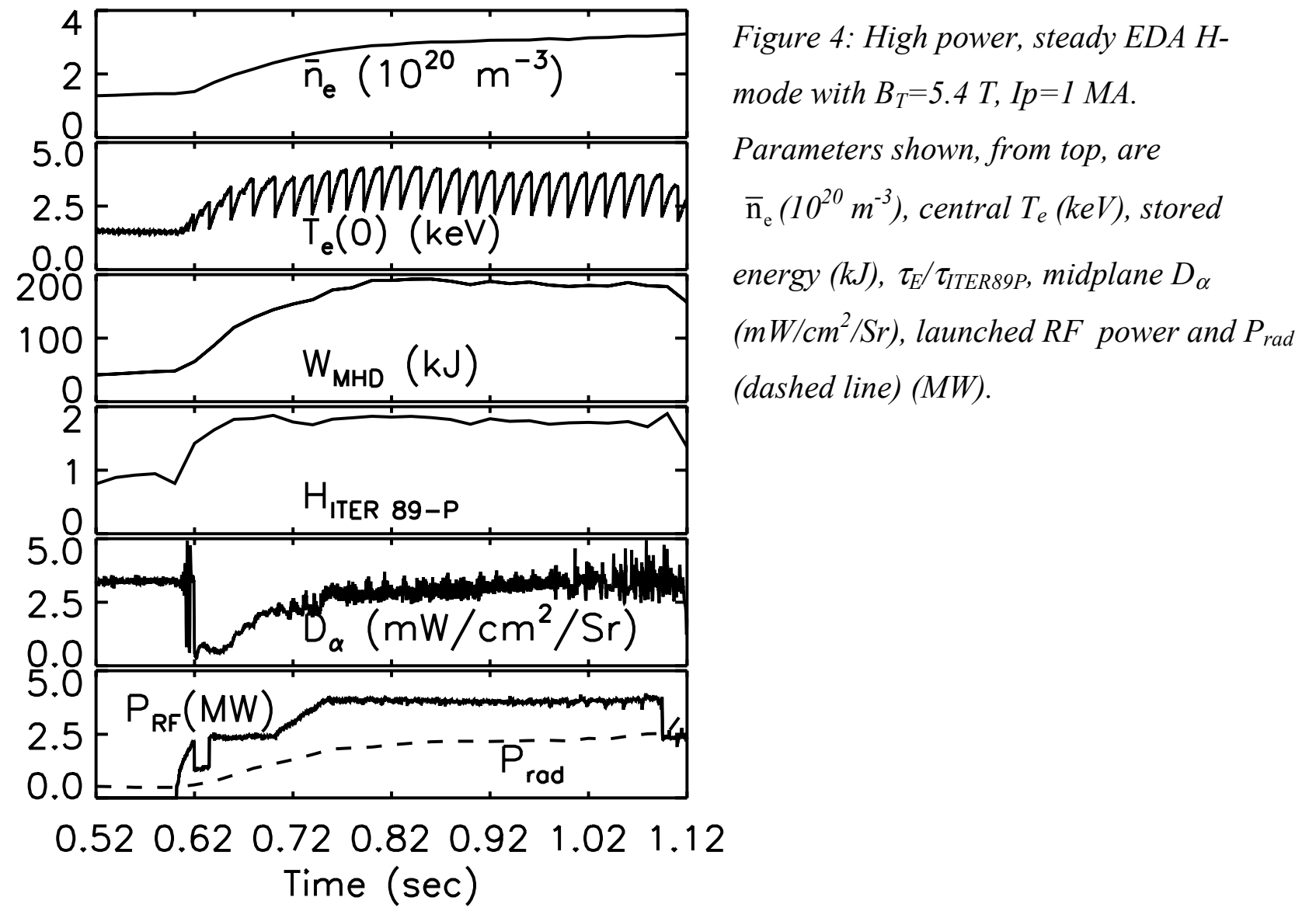

\section{B. Fluctuation Measurements}

Coincident with the observed increases in particle transport in the EDA regime, quasicoherent (QC) edge fluctuations are always observed on several diagnostics. The time evolution of density fluctuation spectra on PCI and reflectometer signals for three short H-modes is shown in Fig. 5. The first H-mode is ELM-free, and shows no feature. In the two EDA H-modes, a peak in the spectra appears, with f initially above $200 \mathrm{kHz}$ and subsequently decreasing to 120 $\mathrm{kHz}$ as the pedestals evolve. The peak frequency in steady EDA is usually in the range of 50-150 $\mathrm{kHz}$. The frequency spectrum, which has $\Delta \mathrm{f} / \mathrm{f} \sim 0.05-0.2$ (full width half maximum), and the wavenumber spectrum, centered at $\mathrm{k}_{\mathrm{R}} \sim 6 \mathrm{~cm}^{-1}$, are measured by PCI. The wavenumber $\mathrm{k}_{\mathrm{R}}$, measured along a major radius at the top and bottom of the plasma, corresponds to a poloidal wavenumber of $\mathrm{k}_{\theta} \sim 4 \mathrm{~cm}^{-1}$. The line integrated amplitude of the density fluctuations is up to $5 \mathrm{x}$ $10^{16} \mathrm{~m}^{-2}$. While the PCI cannot provide spatial localization, this information is obtained from the 
reflectometer and Langmuir probes. The mode is seen near the midplane on reflectometer channels which are located in the steep density region of the pedestal, and does not extend above the barrier. It appears at different density layers depending on $\mathrm{n}_{\mathrm{e}, \mathrm{ped}}$. The transition from the ELM-free to EDA regime is also typically accompanied by an increase in the broadband reflectometrv fluctuations. un to at least $400 \mathrm{kHz}$.

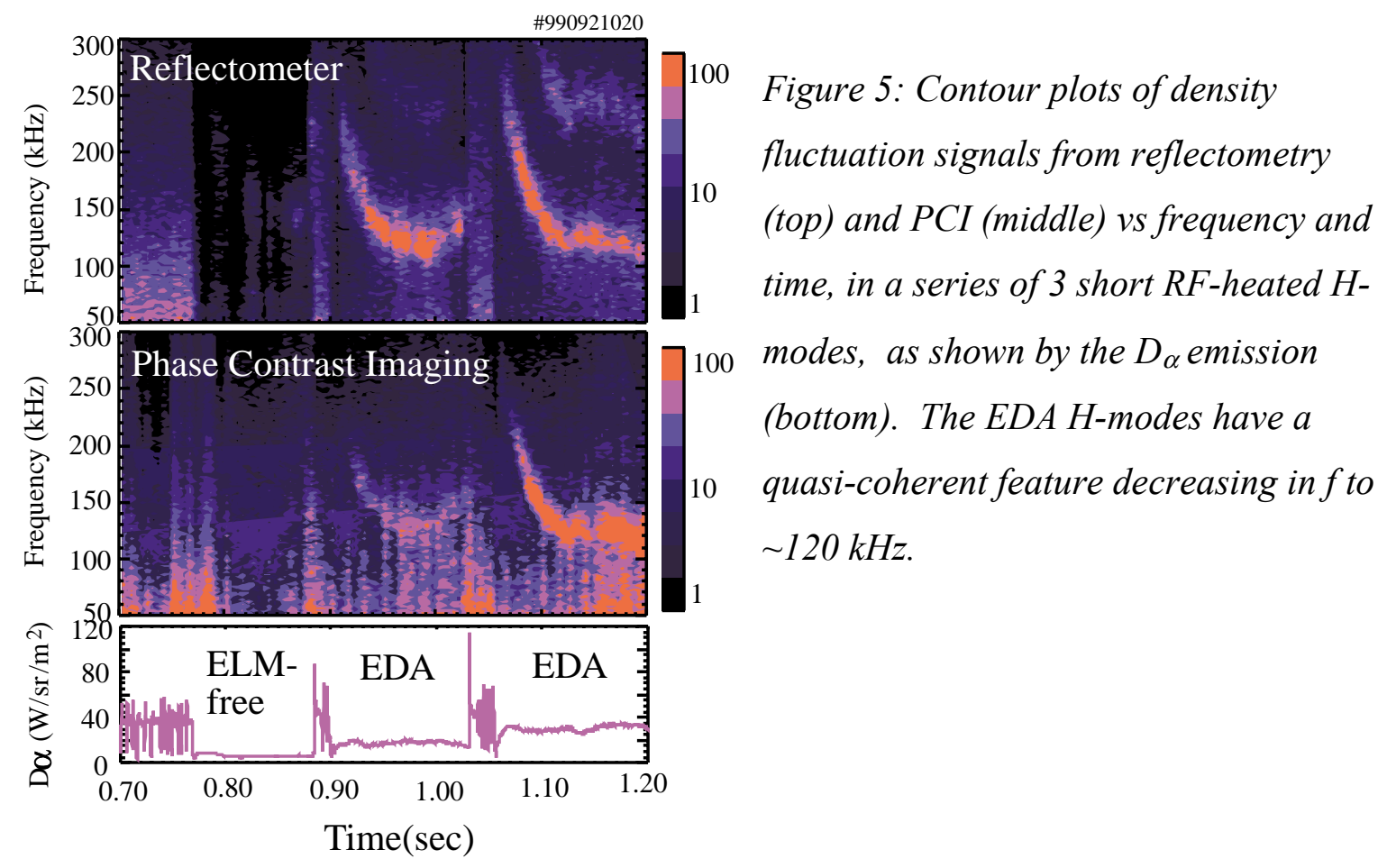

Fast scanning probes, which measure above and below the midplane and have been inserted past the LCFS only in low power ohmic H-modes, also see a strong coherent mode in density and potential at the same frequency. The structure and radial location of the mode are shown in Fig. 6. The amplitude of the fluctuation in potential (6a) only increases once the probe penetrates to within $1 \mathrm{~mm}$ of the separatrix, the same location at which the pedestal density gradient (6b) becomes large. It reaches a strong level, with local fluctuations of order $50 \%$ in both $\tilde{\mathrm{n}}$ and $\tilde{\Phi}$. The amplitude drops $\sim 1 \mathrm{~mm}$ inside $\mathrm{R}_{\text {sep }}$, so that the mode is apparently localized within a region of width $\sim 2 \mathrm{~mm}$. It is possible, however, that the deep penetration of the probe is somehow affecting the fluctuations locally. Direct evidence that the observed quasi-coherent 
fluctuations are indeed responsible for much of the enhanced particle transport is obtained by correlating density and potential fluctuations. The flux $\Gamma$ calculated from $\langle\tilde{n} \cdot \tilde{E}\rangle$, shown in Fig. 6(c), reaches a high level of $1.2 \times 10^{22} / \mathrm{m}^{2} \mathrm{~s}$. In ELM-free H-mode, in contrast, the transported flux is an order of magnitude lower. While there are significant uncertainties associated with such estimates, this strongly suggests that the physics behind the increased particle transport of the EDA regime is linked to the origin of these quasi-coherent fluctuations. In fact, the appearance and strength of the fluctuations is now considered the most reliable signature of the transition between ELM-free and EDA H-modes in either ohmic or RF heated discharges.

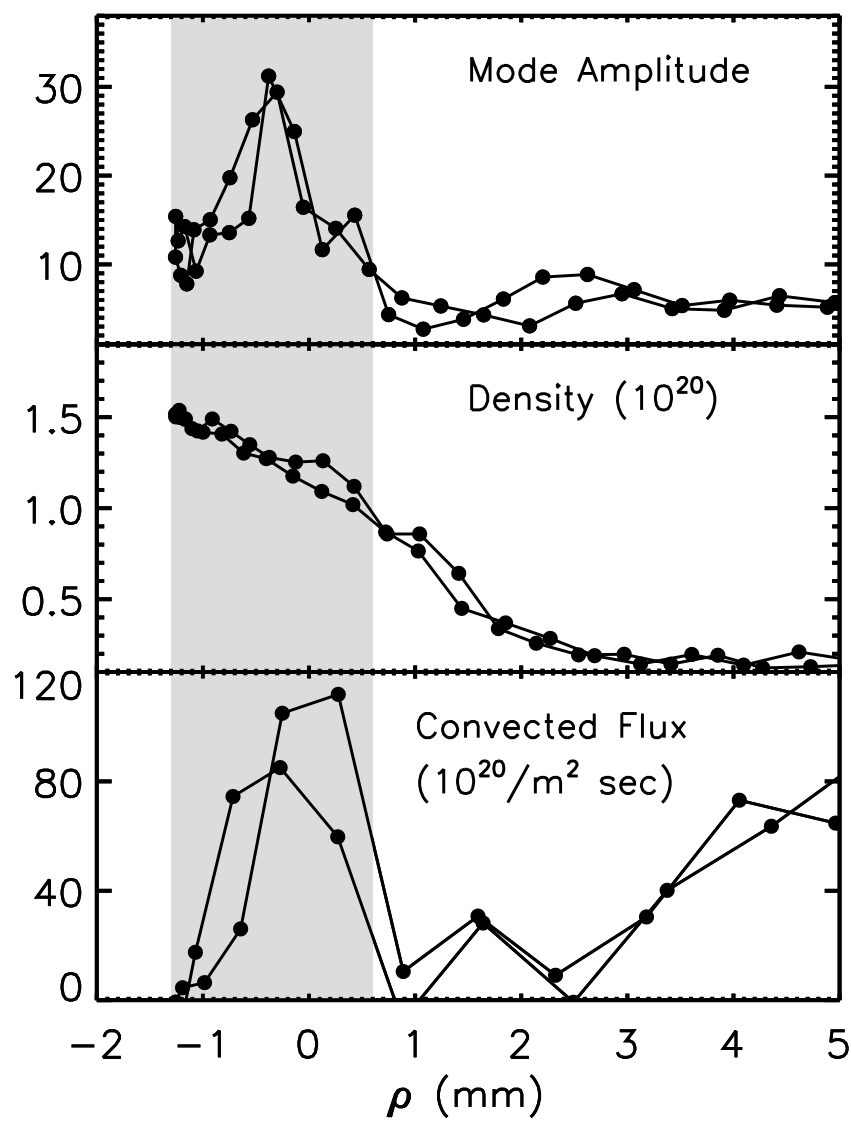

Figure 6: Langmuir probe measurements in an ohmic EDA discharge with $I_{p}=0.72$ $M A$ and $B_{T}=2.6 T$. The two traces are obtained during inward and outward probe scans.

(a) amplitude $\tilde{\Phi}$ of cohererent fluctuations (V),

(b) density profile $\left(10^{20} \mathrm{~m}^{-3}\right)$, (c) particle flux $\Gamma\left(10^{20} / \mathrm{m}^{2} \mathrm{~s}\right)$ derived from $\langle\tilde{\mathrm{n}} \cdot \tilde{\mathrm{E}}\rangle$ for all fluctuation frequencies.

Presumably because of the high $\mathrm{k}$ of the fluctuations, no magnetic signature corresponding to the quasi-coherent mode has been detected on pick-up coils located in the vacuum vessel wall or limiter surfaces. In order to determine if the mode has a magnetic component, a small $\mathrm{B}_{\mathrm{p}}$ probe was mounted on the fast scanning probe head so that it could be moved close to the separatrix. A very clear magnetic signature, up to $200 \mathrm{~T} / \mathrm{s}$, was then seen in the EDA regime. This mode has the same frequency and time evolution as the electrostatic 
fluctuations seen by the reflectometer and Langmuir probe. The peak observed mode amplitude

is $\sim 3 \times 10^{-4} \mathrm{~T}$, corresponding to high local current perturbations, of order $10 \mathrm{~A} / \mathrm{cm}^{2}$ and $\sim 10 \%$ of the edge current density. The QC mode thus has a significant magnetic component. The mode amplitude dropped off very rapidly with radius, typically as $\exp \left(-1.6 \mathrm{~cm}^{-1} \mathrm{r}\right)$, consistent with $\mathrm{k}_{\theta}$ $\sim 1.6 \mathrm{~cm}^{-1}$ and with the lack of signal on limiter coils, which cannot detect levels below $10^{-7} \mathrm{~T}$. In contrast, Type III ELM precursors measured by the scanning magnetic probe during the same discharge series, which had $\mathrm{f} \sim 160 \mathrm{kHz}$, were seen by wall-mounted probes with an amplitude only $\sim 20$ times lower than near $\mathrm{R}_{\text {sep. }}$. This is consistent with a lower $\mathrm{k}_{\theta} \sim 0.5 \mathrm{~cm}^{-1}$, as has previously been reported for ELM precursors ${ }^{27,28}$. The QC mode thus differs from such precursor activity.

\section{Conditions to access the EDA regime}

EDA H-modes have now been obtained routinely on C-Mod over several run campaigns and a wide range of plasma conditions. Systematic studies of the parameters which favour access to the regime have been performed. As has previously been reported ${ }^{24}$, the most consistently important conditions are the safety factor, $q$, and the plasma triangularity, $\delta$. In deuterium plasmas, low $\mathrm{q}_{95}(<3.5)$ and low $\delta(<0.3)$ favour ELM-free discharges. High $\mathrm{q}_{95}(>$ $4.0)$ and higher $\delta(>0.35)$ favour EDA. There may be an upper limit to $\delta$ for EDA, but this operational space has not been well explored. The dependence on elongation, if any, appears to be weak. An ion mass dependence was recently found in experiments with hydrogen plasmas. EDA $\mathrm{H}$-modes were obtained even at $\mathrm{q}_{95}=2.5$, the lowest stable value achieved without disruptions.

EDA H-modes have been obtained both with ICRH and with ohmic heating alone ${ }^{29}$, showing that fast particles do not play a significant role and that high power is not essential. For example, the discharge shown in Fig. 2 transitioned to EDA with power at the LH threshold, and $\overline{\mathrm{n}}_{\mathrm{e}}$ remained in steady state at powers down to the H-L threshold. On the other hand, in some discharges where $\mathrm{q}$ is marginal for EDA, reducing the RF power leads to weaker particle transport and to increasing density and impurity radiation. Ohmic EDA H-modes have been obtained at $\mathrm{B}_{\mathrm{T}}$ as low as $2.5 \mathrm{~T}$ and $\mathrm{I}_{\mathrm{p}}$ down to $0.54 \mathrm{MA}$. 


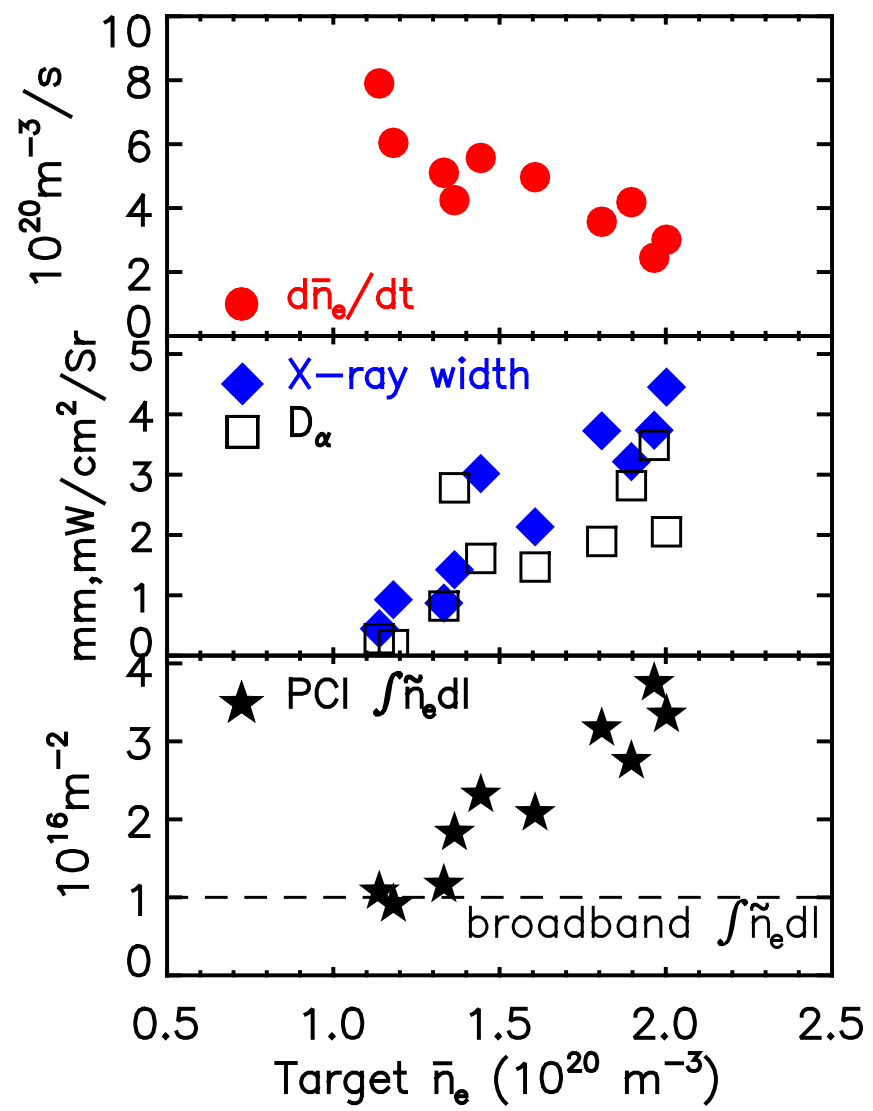

Figure 7: Fluctuations and indicators of particle transport in a series of $5.3 \mathrm{~T}$, 0.8 MA H-modes with varying target density at the L-H transition. H-mode times are taken $80 \mathrm{~ms}$ after the transitions.

(a) $\mathrm{d} \overline{\mathrm{n}}_{\mathrm{e}} / \mathrm{dt}$, which drops as steady state is approached.

(b) $D_{\alpha}$ emission (squares, in $\mathrm{mW} / \mathrm{cm}^{2} / \mathrm{Sr}$ ) and width of the X-ray pedestal (diamonds, in $\mathrm{mm}$ ), which depends on impurity diffusivity.

(c) Amplitude of coherent feature in PCI fluctuations.

A threshold for EDA in target density at the L-H transition is observed ${ }^{30}$. In a series of H-modes (two of which are shown in Fig. 3) with $\mathrm{q}_{95}=4.7$ and $\delta=0.42$, well within the usual EDA range, ELM-free H-modes with no fluctuations were consistently produced when the target $\bar{n}_{\mathrm{e}}$ was less than $1.2 \times 10^{20} \mathrm{~m}^{-3}$. As the density was raised shot to shot, fluctuations appeared and particle transport became progressively stronger. Figure 7 illustrates the gradual transition from ELM-free to EDA transport. Each point corresponds to a different $\mathrm{H}$-mode, with the $\mathrm{H}$-mode time slice taken at $80 \mathrm{~ms}\left(\sim \tau_{\mathrm{E}}\right)$ from the $\mathrm{L}-\mathrm{H}$ transition to avoid the initial transients. The rate of increase of $\bar{n}_{e}(7 a)$ is an indication of deuterium transport. High rates indicate that particles are accumulating rapidly, as is typical of ELM-free discharges, while low rates show an approach to steady state. $\mathrm{dP}_{\text {rad }} / \mathrm{dt}$ (not shown) has a very similar trend. Another measure of impurity transport is the width of the x-ray emissivity pedestal ( $7 \mathrm{~b}$, diamonds), which calculations show depends on $D_{\text {imp }}{ }^{13}$. The $D_{\alpha}$ emission near the midplane is shown for comparison (open squares). The amplitude of the QC density fluctuations on PCI are integrated over $40 \mathrm{kHz}$ about the spectral peak (7c). The dashed line represents the minimum broadband level of fluctuations; points close to this have no visible coherent feature. All of these indicators 
of EDA vary smoothly as $\overline{\mathrm{n}}_{\mathrm{e}}$ is increased from 1.1 to $2.0 \times 10^{20} \mathrm{~m}^{-3}$. In particular, the amplitude of the QC density fluctuation correlates well with all measures of particle transport, giving further evidence that this mode is indeed responsible. This 'soft' threshold for EDA is typical of other boundaries, such as those in q or $\delta$, so that some discharges are difficult to classify as ELM-free or EDA. It should be noted that the threshold density, while higher than typical densities on other tokamaks, is quite low for C-Mod, only $15 \%$ of the Greenwald density limit and close to the low density limit for obtaining H-modes. It is thus not clear how to extrapolate this condition to other devices. The actual threshold is likely to depend on a related quantity, such as the midplane neutral pressure, pedestal density or $\nabla \mathrm{n}$, or edge collisionality. All of these parameters are closely correlated; in particular, the low density ELM-free discharges had $v^{*}<1$ at the pedestal midpoint, whereas more typical higher density H-modes have $v^{*} \sim 5-10$.

\section{Discussion}

H-modes with at least some similarities to the EDA regime have been observed in several other experiments. Quasi-coherent fluctuations which had many similar features to those on CMod were reported in early H-modes on the Princeton Divertor Experiment (PDX) ${ }^{31}$. These tended to occur in short bursts and were apparently seen in most H-modes, including those with ELMs. A 'Low Particle Confinement' regime with global features quite similar to EDA was reported in a few RF heated discharges in the Joint European Torus (JET) ${ }^{32}$. However, recent attempts to reproduce it have not been successful ${ }^{33}$. Probably the most common regime combining small ELMs and good energy confinement is H-mode with Type II or 'Grassy' ELMs. These discharges, which have been observed on DIII-D ${ }^{34}, \mathrm{JT}^{3}-60 \mathrm{U}^{35}$ and most recently on ASDEX Upgrade ${ }^{36}$, are characterized by a decrease in the amplitude and increase in the frequency of (normally Type I) ELMs, often accompanied by a general increase in the $\mathrm{D}_{\alpha}$ level. The conditions for entering this regime, particularly in q and shaping, bear strong similarities to those for EDA; the requirements for density are less clear. However, pedestal fluctuation diagnostics, which could determine whether a QC feature similar to that on EDA was present, were not available for these other experiments. Such measurements would be of great interest to determine whether the Type II and EDA regimes have a common physical origin. The above tokamaks operate at lower field, and typically at higher $\beta_{\mathrm{N}}$, than C-Mod. It should be mentioned that that small ELMs do appear in high power C-Mod discharges when $\beta_{\mathrm{N}}>1$.2. Most recently, 
a 'quiescent' H-mode regime has been reported on DIII-D ${ }^{37}$. It is characterized by continuous density and magnetic fluctuations localized in the pedestal. However, this mode apparently has much longer poloidal wavelength than that seen on C-Mod, and only appears when the edge neutrals are lowered by cryopumping.

Now that detailed measurements of the pedestal profiles and fluctuations in EDA Hmodes are available, work is in progress to identify the physical origin of the mode responsible for the enhanced particle tranport. Since this fluctuation is continuous and at high $\omega$ and $k$, it is likely to involve a microinstability rather than large-scale MHD instability. However, we now know that it has a substantial electromagnetic as well as electrostatic component. Since pedestal parameters and gradients are, somewhat surprisingly, not markedly different in EDA and ELMfree $\mathrm{H}$-modes, it appears that the stability conditions for the mode must be changing; the conditions for EDA thus give some indication of which instability might be involved. Drift ballooning modes are being considered as one possibility. A coherent feature somewhat similar to the QC mode, which has $\mathrm{k}_{\theta} \sim 2 \pi / \mathrm{L}_{\text {ped }}$, has been found in electromagnetic edge turbulence simulations by Rogers and Drake ${ }^{38}$. The condition for diamagnetic stabilization of such modes scales as $\mathrm{m}^{1 / 2} / \mathrm{q}$, so that they become more unstable at high $\mathrm{q}$, consistent with EDA observations. The substantially lower q threshold found for EDA in $\mathrm{H}$ vs $\mathrm{D}$ discharges is also consistent with such a scaling. We plan to study the growth rates of this instability, and its dependence on parameters such as shaping and $v^{*}$, using a gyrokinetic stability code.

While more theoretical and experimental work remains to understand fully the physics of the EDA regime, the results to date show that steady state, high confinement discharges can be reliably and reproducibly produced under a wide range of plasma conditions. The regime appears attractive for fusion reactor applications, provided sufficient plasma shaping is possible. Comparison of EDA with similar regimes on other tokamaks would be very useful in order to build up a database of results under different dimensional conditions and identify which dimensionless parameters, in particular those related to density, might be important.

\section{ACKNOWLEDGEMENTS}

The authors wish to acknowledge the efforts of the entire Alcator C-Mod team, in particular the RF group, in carrying out the experiments reported here. This work was supported by U.S. Dept. of Energy Contract No. DE-FC02-99ER54512 


\section{REFERENCES:}

${ }^{1}$ F. Wagner, G. Becker, K. Behringer et al, Phys. Rev. Letters 49, 1408 (1982).

${ }^{2}$ M Greenwald, R. L.Boivin, F. Bombarda et al, Nucl. Fusion 37 (6), $793-807$ (1997).

${ }^{3}$ W. Suttrop, M. Kaufmann, H.J. de Blank et al, Plasma Phys. Control. Fusion 39 2051-2066 (1997).

${ }^{4}$ A.E. Hubbard, B. Lipschultz, D. Mossessian, R.L. Boivin et al, Proceedings of $26^{\text {th }}$ EPS Conf. on Control. Fusion and Plasma Physics, Maastricht, 1999, edited by B. Schweer, G. Van Oost and E. Vietzke, (European Phys. Soc., 1999), Vol. 23J, p. 13-16.

${ }^{5}$ A. E. Hubbard, Plasma Phys. Contr. Fusion 42 (5A), A15-A36, 2000.

${ }^{6}$ W. Suttrop, Plasma Phys. Contr. Fusion 42 (5A), A1 (2000).

${ }^{7}$ ITER Physics Expert Groups on Divertor, Divertor Modelling and Database and ITER Physics Basis Editors, Nucl. Fusion 39(12) 2391 (1999).

${ }^{8}$ Y. Takase, R. Boivin, F. Bombarda, P. Bonoli et al, Proc. of the $16^{\text {th }}$ Int. Fusion Energy Conf., Montreal, 1996 (International Atomic Energy Agency, Vienna, 1997), Vol. 1, p. 475.

${ }^{9}$ Y. Takase, R. Boivin, F. Bombarda et el, Phys. Plasmas 4 (5), 1647 (1997).

${ }^{10}$ A.E. Hubbard, R.L. Boivin, R.S. Granetz et al, Phys. Plasmas 5 (5), 1744-1751 (1998).

${ }^{11}$ J. W. Hughes, D.A. Mossessian, A.E. Hubbard, E.S. Marmar, D. Johnson and D. Simon, Rev. Sci. Instr. 72 (1) (2001).

${ }^{12}$ E.S. Marmar, R.S. Granetz, J.W. Hughes, B. Lipschultz, S. McCool, D. Mossessian, C.S. Pitcher, J.E. Rice and J.L. Terry, Rev. of Sci. Instr. 72 (1) (2001).

${ }^{13}$ T. Sunn Pedersen, R.S. Granetz, A.E. Hubbard, I.H. Hutchinson, E.S. Marmar, J. E. Rice and J. Terry, Nucl. Fusion 40, 1795 (2000).

${ }^{14}$ R.L. Boivin, J.W. Hughes, B. LaBombard, D. Mossessian and J.L. Terry, Rev. Sci. Instr. 72 (1) (2001).

${ }^{15}$ R. L. Boivin, J.A. Goetz, A.E. Hubbard et al, Phys. Plasmas 7 (5), 1919-1926. (2000)

${ }^{16}$ Y. Lin, J. Irby, P. Stek, I. Hutchinson, J. Snipes, R. Nazikian and M. McCarthy, Rev. Sci. Instrum. 70(1), 1078, (1999).

${ }^{17}$ L.L. Lao, H. St. John, R.D. Stambaugh, A.G. Kellman and W. Pfeiffer, Nucl. Fusion 25, 1611 (1985).

${ }^{18}$ R. J. Groebner and T. N. Carlstrom, Plasma Phys. Control. Fusion 40, 673-678 (1998).

${ }^{19}$ T. Hatae, Y. Kamada, S. Ishida, T. Fukuda, T. Takizuka, H. Shirai, Y. Koide, M. Kikuchi, Y. Yoshida and O. Naito, Plasma Phys. Control. Fusion 40, 1073-1083 (1998).

${ }^{20}$ R.J. Groebner and T.H. Osborne, Phys. Plasmas 5 ,1800-1806 (1998).

${ }^{21}$ W. Suttrop, O. Gehre, J.C. Fuchs et al, Plasma Phys. Control. Fusion 40 771-774 (1998).

${ }^{22}$ R. S. Granetz, T.H. Osborne, R.L. Boivin, M. Greenwald et al, Proc. $17^{\text {th }}$ Fusion Energy Conf., Yokahama, 1998 (International Atomic Energy Agency, Vienna, 1999), Vol. 2, p. 443.

${ }^{23}$ A.E. Hubbard, R. L. Boivin, J. F. Drake, M. Greenwald, Y. In, J. H. Irby, B.N. Rogers and J.A. Snipes, Plasma Phys. Control. Fusion 40, 689-692 (1998).

${ }^{24}$ M. Greenwald. R. Boivin, P. Bonoli et al, Phys. Plasmas 6 (5), 1943-1949 (1999). 
${ }^{25}$ P. Yushmanov, T. Takizuka, K. Reidel, O. Kardaun, J. Cordey, S. Kaye, D. Post, Nucl. Fusion 30, 1999 (1990).

${ }^{26}$ ITER Physics Expert Groups on Confinement and Transport and Confinement Modelling and Database and ITER Physics Basis Editors, Nucl. Fusion 39(12) 2175 (1999).

${ }^{27}$ J.A. Snipes, A. Hubbard, D.T. Garnier et al, Plasma Phys. Control. Fusion 38, 1127-1136 (1996).

${ }^{28}$ I.H. Hutchinson, R.S. Granetz, A. Hubbard et al, Plasma Phys. Control. Fusion 41 , A609 (1999).

${ }^{29}$ M. Greenwald, R. Boivin, P. Bonoli et al, Plasma Phys. Control. Fusion 42 (5A), A263-A269 (2000).

${ }^{30}$ I.H. Hutchinson, R.L. Boivin, F. Bombarda et al, Proc. of the $16^{\text {th }}$ Int. Fusion Energy Conf., Montreal, 1996 (International Atomic Energy Agency, Vienna, 1997), Vol. 1, p. 155.

${ }^{31}$ R.E. Slusher, C.M. Surko, J.F. Valley, T. Crowley, E. Mazzucato, K. McGuire, Phys. Rev. Lett. 53, 667 (1984).

${ }^{32}$ M. Bures, D.J. Campbell, N. Gottardi et al, Nucl. Fusion 32, 539 (1992).

${ }^{33}$ A. Loarte, E. Righi, G. Saibene, R. Sartori, R.D. Monk, P.J. Lomas, G. Maddison, G. Matthews, M. Greenwald, I.H. Hutchinson, C.S. Pitcher and J.A. Snipes, Bull. Am. Phys. Soc. 45(7), 89 (2000).

${ }^{34}$ T. Ozeki, M.S. Chu, L.L. Lao et al, Nucl. Fusion 30, 1425 (1990).

${ }^{35}$ Y. Kamada, T. Oikawa, L. Lao et al, Plasma Phys. Control. Fusion 42, A247-A253 (2000).

${ }^{36}$ J. Stober, O. Gruber. A. Kallenbach et al, Plasma Phys. Control. Fusion 42, A211-A216 (2000).

${ }^{37}$ K.H. Burrell, M.E. Austin, D.P. Brennan et al, submitted for publication in this issue.

${ }^{38}$ B. Rogers and J. Drake, Phys. Plasmas 6, 2797 (1999). 